\title{
Communication
}

\section{In-Situ Assessment of PAHs in Contaminated Sea Sediments}

\author{
Emanuela Frapiccini ${ }^{1}$, Evgeniya Prokofyeva ${ }^{2,3}$, Antonina Bondarenko ${ }^{3}$, \\ Maria Letizia Ruello ${ }^{2}$ and Mauro Marini ${ }^{1, *}$
}

1 CNR-ISMAR, largo Fiera della Pesca, 2, 60125 Ancona, Italy; emanuela.frapiccini@an.ismar.cnr.it

2 Dipartimento di Fisica e Ingegneria dei Materiali e del Territorio, Università Politecnica delle Marche,

SIMAU Via Brecce Bianche, 60131 Ancona, Italy; e.prokofyeva@univpm.it (E.P.); m.1.ruello@univpm.it (M.L.R.)

3 Department of Chemical Technology, Lipetsk State Technical University, Moskovskaya st., 30,

398055 Lipetsk, Russia; antonina.bondarenko@gmail.com

* Correspondence: m.marini@ismar.cnr.it

\begin{abstract}
The present study describes the activities performed to test a new method for measuring the mobility of polycyclic aromatic hydrocarbons (PAHs) in the solid phase of sediments within the context of environmental pollution risk assessment. The method is based on the design of a new configuration (new materials) of the commercial passive sampler Chemcatcher as probe for predicting the bioavailability of persistent organic pollutants in marine sediments (or in water saturated soils).
\end{abstract}

Keywords: persistent organic pollutants, bioavailability, Diffusive Gradient Thin film (DGT), passive probe, degradation

\section{Introduction}

The DGT-technique (Diffusive Gradient Thin film) is a passive sampling technique, which has been successfully applied for measuring inorganic contaminants in water, sediments and soils [1,2]. In the present work this principle has been used for PAHs evaluation in sediment. The research showed the developing of the method for environmental risk assessment of contaminated sediments by using adsorptive equilibrium of pollutant in system solid matrix-water solution. The technique was designed by adapting the DGT method, originally meant for heavy metals [3], to organic molecules. This method is able to measure PAHs concentration in sediments by perturbing the equilibrium between solid and liquid phases after diffusion through a thin deionized water layer.

\section{Materials and Methods}

\subsection{Study area, Sampling}

Two marine sediments were collected from Senigallia transect in Adriatic Sea (Mediterranean Sea): the site 1 (open sea, $44^{\circ} 02.96 \mathrm{~N} \mathrm{13}{ }^{\circ} 41.03 \mathrm{E}$ ) and the site 7 (coastal, $43^{\circ} 45.87 \mathrm{~N} 13^{\circ} 13.03 \mathrm{E}$ ). These sediments are classified as clay loam and sandy clay loam, respectively, as agreement by Shepard's scale [4]. The total organic carbon (TOC) content resulted similar for both sites $(0.3 \%)$. Shallow sediment samples $(0-2 \mathrm{~cm})$ of both study sites were collected using a box corer. Cleaned sediment samples were stored in closed jars of transparent glass, in the presence of oxygen and at $4 \pm 2{ }^{\circ} \mathrm{C}$. 


\subsection{Sampler design}

The passive sampling device consisted of a C18 Empore disc as chromatographic receiving phase overlaid with a Whatman glass microfiber filter membrane (47 $\mathrm{mm}$ diameter). The water is added to the interstitial space between the receiving phase and membrane. The principle of measurement for the probe is based on Fick's first law as the traditional diffusion-adsorption technique [5]. The receptor material was separated from the specimen by a water chamber of thickness $\Delta \mathrm{g}(0,2 \mathrm{~mm})$. This technique is named DGT because the gradient of concentrations produced by the water chamber of thickness $0,2 \mathrm{~mm}$ is the driving force for diffusion. Organic molecules diffuse through the water layer and are rapidly bound by the receptor material, so their concentration at the interface waterreceptor is maintained at zero throughout the deployment. Water chamber and glass fiber membranes were chosen specifically in order not to interpose selective media for the pollutant diffusion process $[6,7]$. The probe measures the average flux of pollutant that diffuses through the water layer by accumulating a mass of pollutant over time through a well-defined area. In the DGT technique, solutes are dynamically removed by their diffusion through a membrane to a binding adsorbent material, so the sampled PAHs represent the labile pollutants pools. The PTFE Chemcatcher body [8] supported both the receiving phase and the diffusion membrane and sealed them in place.

\subsection{Preparation of the samplers}

C18 Empore disks were conditioned by soaking them in methanol for 20 min until translucent and then stored in methanol until required. The Empore disks were prepared in a $47 \mathrm{~mm}$ diameter disk vacuum manifold platform. Methanol $(10 \mathrm{~mL})$ was slowly passed through the disk, followed by 10 $\mathrm{mL}$ ultrapure water. The conditioned disks were placed in the Chemcatcher body, which was subsequently filled with purified water. The glass microfiber filter membrane was put on the top of the Empore disk. Any air bubbles were smoothed away from between the two layers by gently pressing the top surface of the membrane. The PTFE supporting disk was placed into the sampler body and fixed in place to form a watertight seal between the membrane and the top section of the sampler.

\subsection{PAH extraction for bio/degradation parameters}

The standard PAH solution EPA 610 was used as inoculum for the batch degradation test. The collected sediments (about $200 \mathrm{~g}$ ) were spiked with a diluted standard PAHs solution $(5971.2 \mu \mathrm{g} / \mathrm{L}$ ). A known quantity of $40 \%$ formalin $(200 \mu \mathrm{L})$ has been added to each sediment samples in order to observe in parallel the degradation kinetic without microbial activity. Both bio/degradation rates were studied after equilibration of one day for a period of 49 days. The extractions were conducted in prefixed time periods after inoculation and equilibration. For both degradation studies the U.S. EPA's 16 priority PAH were extracted from $10 \mathrm{~g}$ wet sediment samples. The PAH extraction from sediment was achieved by three 15 min cycles in an ultrasonic baths [9] using as solvent methanol:dichloromethane (1:1) and a liquid-liquid separation was made. The PAH enriched solvent removed initially by rotary evaporation $\left(\mathrm{T}=30 \pm 2{ }^{\circ} \mathrm{C}\right)$ and later by gentle nitrogen flow [10]. The final volume of the analytical sample was adjusted to $300 \mu \mathrm{L}$ with acetonitrile.

PAH quantification and PAH qualification were carried out by high performance liquid chromatography (HPLC Ultimate 3000, Thermo Scientific, USA) with fluorescence and diode array 
deterctors (Thermo Scientific, USA) [11]. Calibration solutions were prepared by serial dilutions from the standard PAH solution.

The wet weight of each sample of marine sediment was corrected to the dry weight, after the determination of the percentage of humidity in the sediment samples. Each concentration was expressed on a dry-weight basis.

\subsection{Extraction of analytes form the passive samplers}

After exposure, the sampler was carefully disassembled and the membrane removed. Compounds were extracted from the Empore disks in an ultrasonic bath $(5 \mathrm{~min})$ using acetone $(5 \mathrm{~mL})$ followed by $5 \mathrm{~min}$ in $50: 50(\mathrm{v} / \mathrm{v})$ ethyl acetate: 2,2,4-trimethylpentane $(5 \mathrm{~mL})$. The disks were removed and the solvent extracts combined. The solvent extract was gradually reduced under nitrogen. The reduced extract was transferred to $2 \mathrm{~mL}$ vials prior to analysis. The final volume was adjusted to $300 \mu \mathrm{L}$ with acetonitrile. PAH quantification were carried with the same method described by Marini and Frapiccini [11].

\section{Results and Discussion}

At the both sites almost all the target pollutants were found with the used passive sampler; but only with the exception of the naphthalene because it may be lost from samples via evaporation. In our ongoing work we present calculation only for anthracene, pyrene and benzo(a)pyrene. The issues that will be addressed in the further validation of the passive sampler include testing the other PAHs from these experiments.

\subsection{Calculation of $C_{D G T}$ concentration and ratio $R$}

The passive sample device continuously accumulates pollutants in the receiving phase. After retrieval, the total mass of the pollutant $(M)$ in the layer is measured. The time-averaged interfacial concentration (CDGT) can be calculated from $M$ and the deployment time $(\mathrm{t})$ :

$$
C_{D G T}=\frac{M \cdot s}{A \cdot t \cdot D}
$$

eq. 1

where $\mathrm{D}$ is the diffusion coefficient $\left(\mathrm{cm}^{2} / \mathrm{s}\right)$ and $\mathrm{A}$ is the exposed area of diffusion chamber of thickness.

The concentrations of PAHs as perceived by the passive sampler considered in the work is compared with the measured concentration in the pore water. Comparison of CDGT with the independently measured concentration in solution $\left(\mathrm{C}_{\mathrm{ss}}\right)$ provides a ratio $(R)$ that indicates the extent of the depletion of solution concentration at the device interface:

$$
R=\frac{C_{D G T}}{C_{s s}}
$$

The trends in time of $R$, for the analysed PAHs in both sediments, are shown in Figure 1. 

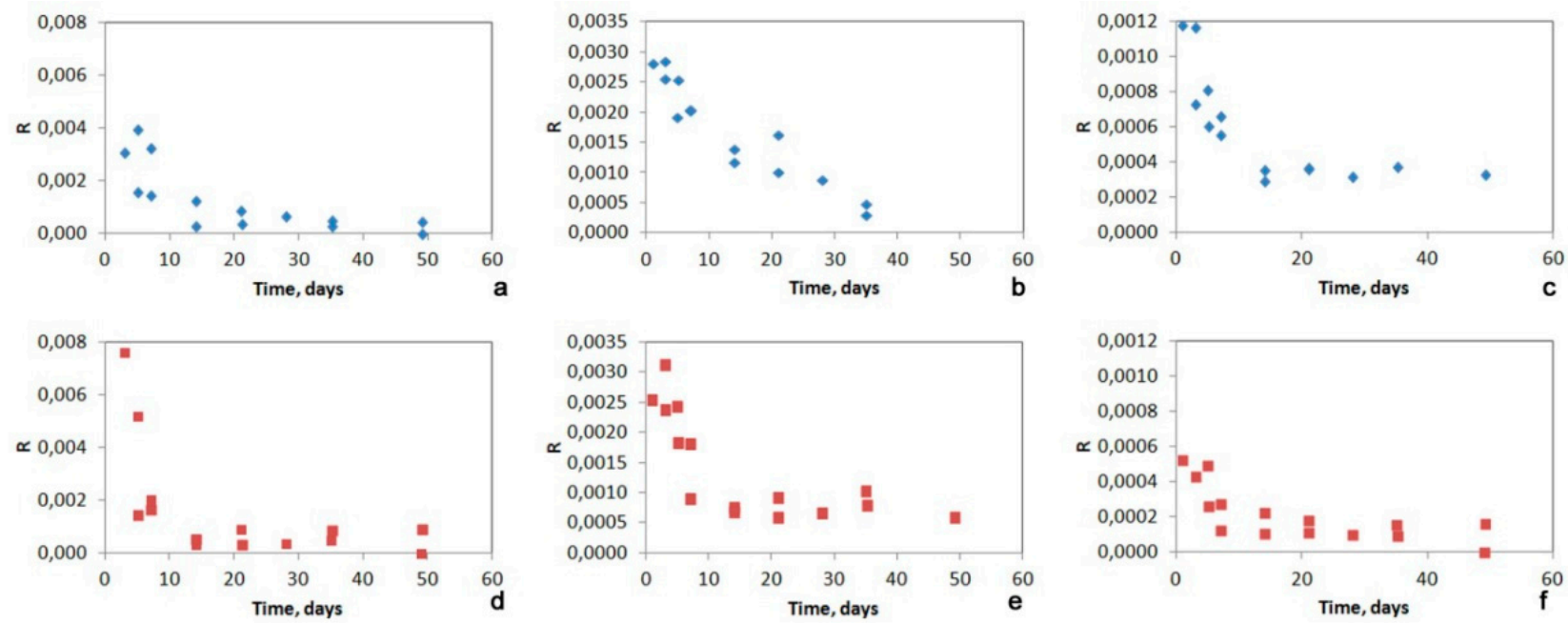

Fig. 1: Ratio (R) between concentration of anthracene $(a, d)$, pyrene $(b, e)$ and benzo(a)pyrene $(c, f)$ measured by passive probe and measured in the pore water for site $1(a, b, c)$ and site $7(d, e, f)$.

The graphs show that the ratio $(R)$ between these concentrations quickly decreases in the first 2 weeks and then remains constant at very low values. This behaviour is indicative of the low ability of the solid phase in sustain the pore water concentration. After 14 days this ability is so low that the concentration in solution is sustained only by diffusion and no more by resupply from the solid phase. This results are supported by the high half-life of benzo(a)pyrene measured by the degradation test. The higher value of $R$ is obtained for anthracene, and this value decreases for the PAHs with 4 and 5 rings. Furthermore, for more clayey marine sediments (site 1 ) the $\mathrm{R}$ values is higher. This is indicative of a greater amount of PAHs adsorbed in solid phase in a labile form, this labile pool restores the pore water concentration after the depletion by the probe uptake.

\subsection{Processes of bio/degradation of PAHs in marine sediments}

The PAHs analyzed in site 1 showed greater half-lives in comparison with site 7 (Table 1).

Table 1. Half-lives (days) of anthracene, pyrene and benzo(a)pyrene in the central Adriatic marine sediments (site 1 and site 7) with and without microbial activity and the differences between two half-lives for each PAH.

\begin{tabular}{lcccccccc}
\hline \multirow{2}{*}{ PAHs } & \multicolumn{3}{c}{ Site 1 } & & \multicolumn{3}{c}{ Site 7 } \\
\cline { 2 - 3 } \cline { 8 - 9 } & $\begin{array}{c}\text { with } \\
\text { m.a. }\end{array}$ & $\begin{array}{c}\text { without } \\
\text { m.a. }\end{array}$ & difference & & $\begin{array}{c}\text { with } \\
\text { m.a. }\end{array}$ & & without & difference \\
m.a. & \\
\hline Anthracene (3 rings) & 66.0 & 100.4 & 34.4 & & 29.6 & 84.5 & 54.9 \\
Pyrene (4 rings) & 85.6 & 141.4 & 55.8 & & 62.4 & 108.3 & 45.9 \\
Benzo(a)pyrene (5 rings) & 161.2 & 187.3 & 26 & & 90.0 & 157.5 & 67 \\
\hline
\end{tabular}

m.a. = microbial activity

PAHs sorption ability of a marine sediment is affected by its particle size and primarily by TOC content [9]. As the TOC content is similar for both sites, the rate of degradation is affected by metabolic activity of the microorganisms. The lack of the metabolic activity of the microorganisms in the studied marine sediment (site 1) has lowered PAH degradation. Therefore, high molecular weight (HMW) PAHs as benzo(a)pyrene have recorded a greater difference of half-lives between two conditions (presence/absence microorganisms) mostly in the coastal sediment (site 7) in comparison 
with open sea one (site 1). This is due to fact that the biodegradation of organic compounds is facilitated by the presence of environmental conditions more favourable to microorganisms in the coastal sediment.

\section{Conclusions}

The present study describes the activities performed to test a new method for measuring the mobility of PAHs in the solid phase of sediments within the context of environmental pollution risk assessment. The method is based on the design of a new probe for the passive sampling of organic pollutant in sediments (or in water saturated soils). The results show that the adopted configuration of the passive probe (glass microfiber filter membrane and C18 Empore disk) is able to measure the labile fraction of PAHs in marine sediments. This information is very useful to predict the fate of the pollutants in the environment. In fact, the presence of a labile fraction of pollutant makes possible its degradation when bacteria activity exists.

\section{Acknowledgments}

We thank The Ministry of Education and Science of Russia for the program of mobility funding that supports the joint $\mathrm{PhD}$ degree between Lipetsk State Technical University and Università Politecnica delle Marche. This research is supported by the Bandiera RITMARE Project coordinated by National Research Council and financed by Italian University and Research Ministry, National Research Program, 2011-2013.

\section{References}

[1] Davison, W.; Zhang, H. In situ speciation measurements of trace components in natural waters using thin-film gels. Nature 1994, 367, 546-548.

[2] Davison, W.; Zhang, H. Progress in understanding the use of diffusive gradients in thin films (DGT)-back to basics. Environm. Chem. 2012, 9, 1-13.

[3] Ruello, M.L.; Sileno, M.; Sani, D.; Fava, G. DGT use in contaminated site characterization. The importance of heavy metal site specific behaviour. Chemosphere 2008, 70, 1135-1140.

[4] Shepard, F.P. Nomenclature based on sand-silt-clay ratios. J. Sediment. Petrol. 1954, 24, 151158.

[5] Zhang, H.; Davison, W.; Knight, B., McGrath, S. In Situ measurements of solution concentrations and fluxes of trace metals in soils using DGT. Environ. Sci. Technol. 1998, 32, 704-710.

[6] Kingston, J.K.; Greenwood, R.; Mills, G.A.; Morrison, G.M.; Persson, L.B. Development of a novel passive sampling system for the time-averaged measurement of a range of organic pollutants in aquatic environments. J. Environ. Monit. 2000, 2, 487-495.

[7] Bondarenko, A.; Sani, D.; Ruello, M.L. Design and calibration of an organic diffusive probe to extend the diffusion gradient technique to organic pollutants. Int. J. Environ. Res. Public Health 2011, $8,3318-3332$. 
[8] Vrana, B.; Mills, G.A.; Allan, I.J.; Dominiak, E.; Svensson, K.; Knutsson, J.; Morrison, G.; Greenwood, R. Passive sampling techniques for monitoring of pollutants in water. Trends Analyt. Chem. 2005, 24, 845-868.

[9] Marini, M.; Frapiccini, E. Do lagoon area sediments act as traps for polycyclic aromatic hydrocarbon? Chemosphere, 2014, 111, 80-88.

[10] Barret, M.; Carrère, H.; Latrille, E.; Wisniewski, C.; Patureau, D. Micropollutant and sludge characterization for modeling sorption equilibria. Environ. Sci. Technol. 2010, 44, 1100-1106.

[11] Marini, M.; Frapiccini, E. Persistence of polycyclic aromatic hydrocarbons in sediments in the deeper area of the Northern Adriatic Sea (Mediterranean Sea). Chemosphere 2013, 90, 1839-1846.

(C) 2016 by the authors; licensee Preprints, Basel, Switzerland. This article is an open access article distributed under the terms and conditions of the Creative Commons by Attribution (CC-BY) license (http://creativecommons.org/licenses/by/4.0/). 